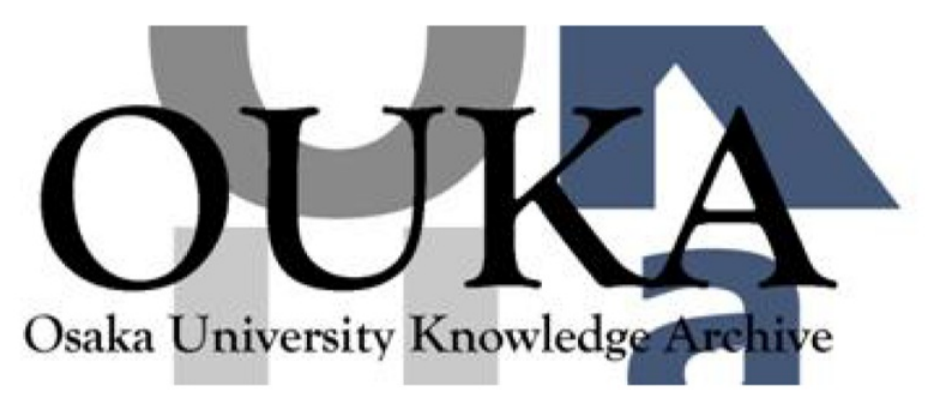

\begin{tabular}{|c|l|}
\hline Title & $\begin{array}{l}\text { Fabrication and characteristics of } 8- \\
\text { hydroxyquinoline aluminum/aromatic diamine } \\
\text { organic multiple quantum well and its use for } \\
\text { electroluminescent diode }\end{array}$ \\
\hline Author(s) & $\begin{array}{l}\text { Ohmori, Yutaka; Fuji, Akihiko; Uchida, Masao } \\
\text { et al. }\end{array}$ \\
\hline Citation & Applied Physics Letters. 62(25) p. 3250-p. 3252 \\
\hline Issue Date & $1993-06-21$ \\
\hline oaire:version & VoR \\
\hline URL & https://hdl.handle.net/11094/75648 \\
\hline rights & \\
\hline Note & \\
\hline
\end{tabular}

Osaka University Knowledge Archive : OUKA

https://ir. Library. osaka-u. ac. jp/

0saka University 


\section{Fabrication and characteristics of 8- hydroxyquinoline aluminum/aromatic diamine organic multiple quantum well and its use for electroluminescent diode}

Cite as: Appl. Phys. Lett. 62, 3250 (1993); https://doi.org/10.1063/1.109089

Submitted: 09 December 1992 . Accepted: 30 March 1993 . Published Online: 04 June 1998

Yutaka Ohmori, Akihiko Fujii, Masao Uchida, Chikayoshi Morishima, and Katsumi Yoshino

\section{ARTICLES YOU MAY BE INTERESTED IN}

Organic electroluminescent diodes

Applied Physics Letters 51, 913 (1987); https://doi.org/10.1063/1.98799

Observation of spectral narrowing and emission energy shift in organic

electroluminescent diode utilizing 8-hydroxyquinoline aluminum/aromatic diamine multilayer structure

Applied Physics Letters 63, 1871 (1993); https://doi.org/10.1063/1.110632

Quasi-epitaxial growth of organic multiple quantum well structures by organic molecular beam deposition

Applied Physics Letters 56, 674 (1990); https://doi.org/10.1063/1.102733

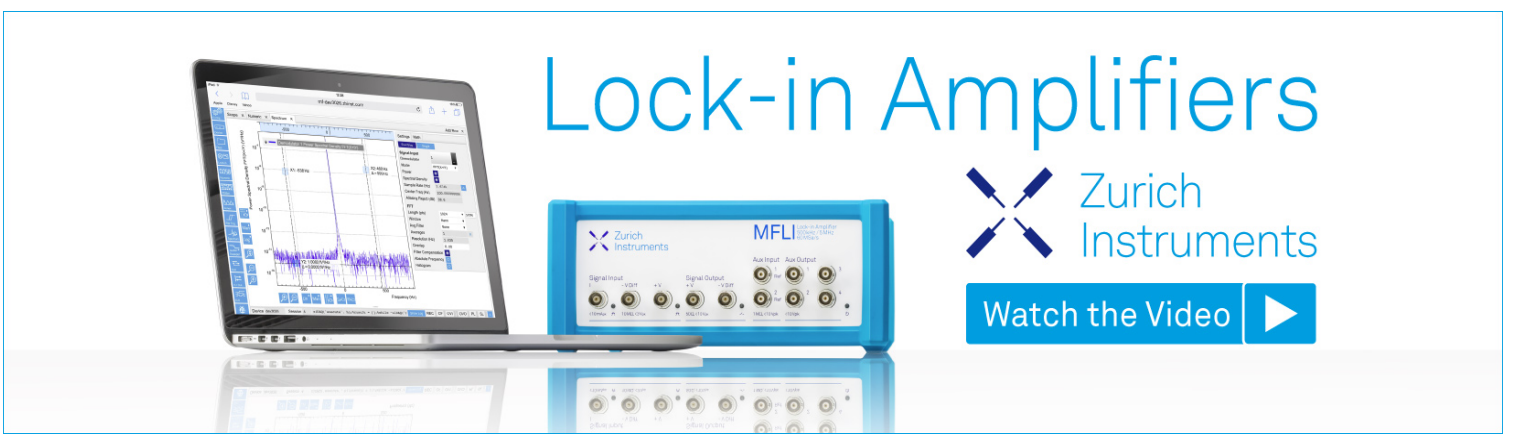




\title{
Fabrication and characteristics of 8-hydroxyquinoline aluminum/aromatic diamine organic multiple quantum well and its use for electroluminescent diode
}

\author{
Yutaka Ohmori, Akihiko Fujii, Masao Uchida, Chikayoshi Morishima, \\ and Katsumi Yoshino \\ Faculty of Engineering, Osaka University, Yamada-oka, Suita, Osaka 565, Japan
}

(Received 9 December 1992; accepted for publication 30 March 1993)

\begin{abstract}
Multiple quantum well structure consisting of alternating layers of organic 8-hydroxyquinoline aluminum $\left(\mathrm{Alq}_{3}\right)$ and aromatic diamine (TPD) has been grown by organic molecular beam deposition. The multiple quantum well structure was determined by $x$-ray diffraction, optical absorption, and photoluminescence. Photoluminescence peak of $\mathrm{Alq}_{3}$ shifts to higher energy with decreasing layer thickness, suggesting a quantum size effect. An electroluminescent diode has also been fabricated by using $\mathrm{Alq}_{3}$ /TPD multiple quantum well structure.
\end{abstract}

Organic electroluminescent diode $\mathrm{e}^{1-7}$ has attracted much interest because of its potentiality in material and device process. Tang et al. ${ }^{1}$ developed a very efficient fluorescent material $\left(\mathrm{Alq}_{3}\right)$ and demonstrated low-voltage driven electroluminescent (EL) diodes using $\mathrm{Alq}_{3}$ as an emitting material. EL diodes with conducting polymers ${ }^{3-7}$ have also been developed and reported.

On the other hand, inorganic semiconductor superlattices ${ }^{8}$ and multiple quantum wells (MQWs) have substantially developed, and laser diodes with MQW structure $e^{9,10}$ of III-V compound semiconductors have been developed. The MQWs and the laser diodes have demonstrated many unique optical and electrical characteristics compared with conventional bulk materials.

Recently, So et al. ${ }^{11,12}$ reported fabrication and characteristics of crystalline organic MQWs by organic molecular beam deposition. Organic EL diode with a MQW structure will be expected to have narrow spectral emission, the improvement of emission efficiency, and even tunable emission spectrum, in the future.

In this letter, we report fabrication and characteristics of the multilayer structure of 8-hydroxyquinoline aluminum [tris (8-hydroxyquinoline) aluminum, $\mathrm{Alq}_{3}$ ] and aromatic diamine $\left\{N, N^{\prime}\right.$-diphenyl- $N, N^{\prime}$-bis (3methylphenyl)-[1,1'-biphenyl]-4,4'-diamine, TPD\}. The characteristics of the MQW structure are determined by $\mathrm{x}$-ray diffraction, optical absorption, and photoluminescence. The electroluminescent diodes with the organic MQW structure have been fabricated and the emission characteristics are discussed. Quantum size effect of the MQWs has also been discussed.

Organic multilayer structure was grown onto two kinds of substrates. The quartz substrate was used for optical measurement and the indium-tin oxide (ITO)-coated glass substrate for the EL diode, which was fabricated by organic molecular beam deposition. The base chamber pressure was under $10^{-6}$ Torr. The powders of $\mathrm{Alq}_{3}$ and TPD were loaded to each separate Knudsen cell. The cells were subsequently heated up to sublimate at a growth rate of about $0.1 \mathrm{~mm} / \mathrm{s}$ which was determined by an oscillating quartz thicknesses monitor. A series of multilayer samples consists of an alternate layer of $\mathrm{Alq}_{3}$ and TPD, whose layer thicknesses were changed from 1.9 to $27.1 \mathrm{~nm}$. The layer structure of the MQW samples was determined by the $\mathrm{x}$-ray diffraction ( $\mathrm{Cu} K \alpha$ line). Optical absorption and photoluminescence were measured at room temperature using conventional methods.

The structure of a multilayer sample which consists of alternating layers of $\mathrm{Alq}_{3}$ and TPD with the same thickness was determined by $\mathrm{x}$-ray diffraction. $\mathrm{Alq}_{3}$ and $\mathrm{TPD}$ layers deposited on substrates are reported microcrystalline (about $50 \mathrm{~nm}$ crystal size) ${ }^{13}$ and noncrystalline, respectively. We have also observed no featured diffraction pattern from the deposited film in the diffraction angle $2 \theta$ from $2^{\circ}$ to $60^{\circ}$. Nevertheless we have observed the diffraction pattern in the low angle position, which corresponds to the signal of the multilayer structure. The $\mathrm{x}$-ray diffraction pattern from the multilayer of $\mathrm{Alq}_{3}$ /TPD corresponds to the signal which was reported for multilayered thin-film semiconductors, such as GaAs/AlAs thin-film multilayers. ${ }^{14}$ We only observed the $\mathrm{x}$-ray diffraction signal from the multilayer thin films, but not from the single layer film. This fact shows that the diffraction from the multilayer structure with the organic solid can also be obtained. In Fig. 1, a typical x-ray diffraction pattern is shown for a multilayer with the total thickness of $136 \mathrm{~nm}$ ( 15 periods of each $4.6 \mathrm{~nm} \mathrm{Alq}$ and TPD layers). The diffraction pattern which is indicated by an arrow corresponds to the satellite peak of the monolayer period and the other peri-

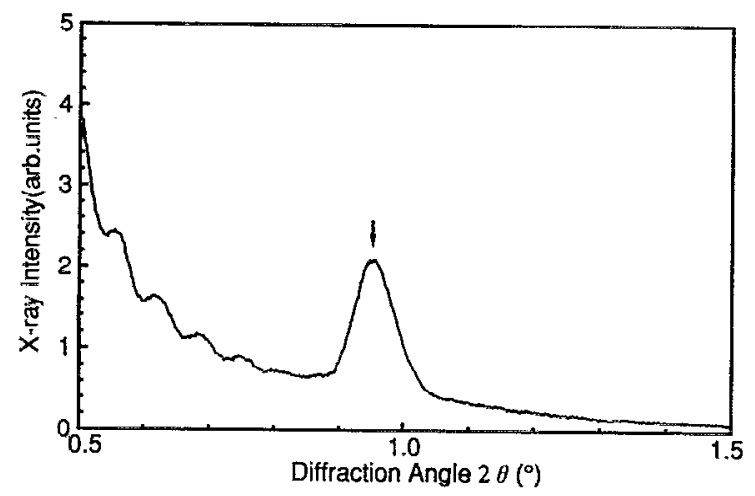

FIG. 1. X-ray diffraction pattern of $\mathrm{Alq}_{3} / \mathrm{TPD}$ multilayer. 


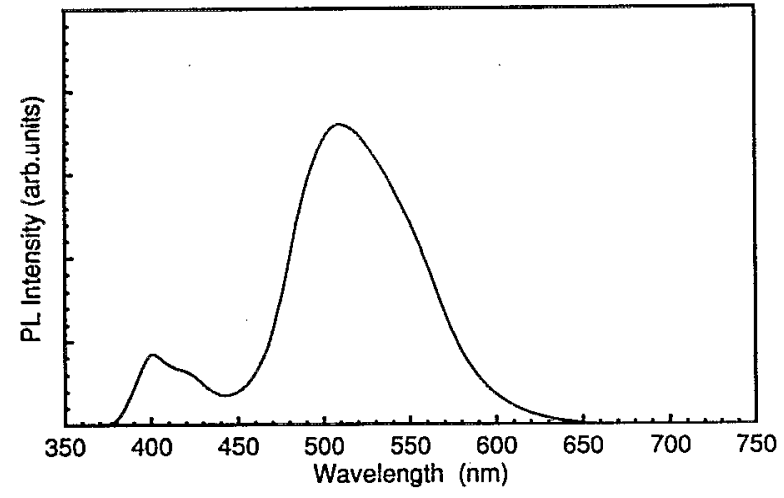

FIG. 2. Photoluminescence spectrum of $\mathrm{Alq}_{3} / \mathrm{TPD}$ multilayer. The MQW structure consists of $\mathrm{Alq}_{3}$ and TPD with cach $10.9 \mathrm{~nm}$ layer thickness.

odical diffraction peaks correspond to the total layer. In the low angle side, the leakage beam from a direct $x$-ray source are superimposed to the original diffraction pattern.

Photoluminescence measurement has been done for the samples with a multilayer structure. The multilayer samples exhibit strong fluorescence from the $\mathrm{Alq}_{3}$ layer (at around $510 \mathrm{~nm}$ ) and rather weak emission from the TPD layer (at around $400 \mathrm{~nm}$ ) as shown in Fig. 2. The emission peak, which is originated from the $\mathrm{Alq}_{3}$ layer, shifts to higher energy according to the decrease in layer thickness of the $\mathrm{Alq}_{3}$. The energy gaps of $\mathrm{Alq}_{3}$ and TPD were obtained from optical absorption edge, and are estimated as 2.6 and $3.2 \mathrm{eV}$, respectively. The ionization potential of the $\mathrm{Alq}_{3}$ and TPD have been determined by photoelectron emission spectroscopy and was reported as -5.7 and -5.4 $\mathrm{eV}$, respectively. Therefore, the energy band of the MQW has been found to be the type I' superlattice as shown in the inset of Fig. 3. Electrons in an $\mathrm{Alq}_{3}$ layer are sandwiched by the TPD energy barriers, and the holes in TPD layer by the $\mathrm{Alq}_{3}$, separately. The energy barricr for the electrons in the $\mathrm{Alq}_{3}$ layer is estimated as $0.9 \mathrm{eV}$, whereas the barrier for the holes in TPD is $0.3 \mathrm{eV}$. Quantized energy levels are indicated schematically in the dashed line in the figure. Since the conduction type of $\mathrm{Alq}_{3}$ is reported to be $n$-type, ${ }^{1}$ electrons in $\mathrm{Alq}_{3}$ are localized by the TPD

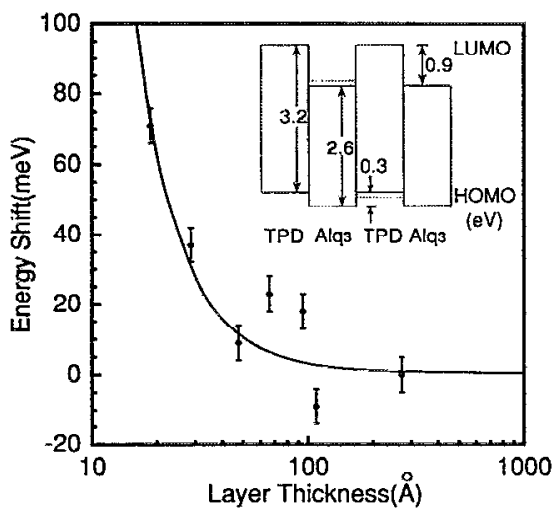

FIG. 3. Energy shift in photoluminescence peak in $\mathrm{Alq}_{3}$ /TPD multilayer. Inset shows energy band diagram of $\mathrm{Alq}_{3} / \mathrm{TPD}$ multilayer structure.

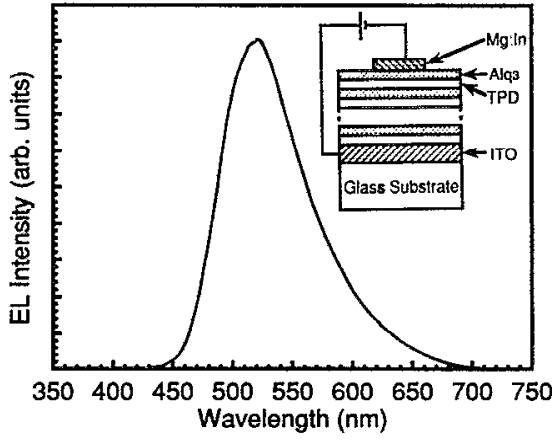

FIG. 4. Electroluminescence spectrum of the diode with $\mathrm{Alq}_{3} / \mathrm{TPD}$ multilayer. Inset shows cross sectional view of $\mathrm{EL}$ diode with $\mathrm{Alq}_{3} / \mathrm{TPD}$ multilayer structure.

barrier. This is consistent with the fact that the peak emission from $\mathrm{Alq}_{3}$ shifts to higher energy according to the decrease in layer thickness. The holes in the TPD layer should also be localized but the energy shift has not been confirmed due to the weak and broad emission band.

The energy shift is evaluated using the Kronig-Penny model for the localized electrons. In the calculation, we used infinite barrier height and the effective electron mass $m_{e}^{*}$ as a parameter. As shown in Fig. 3, the calculated value of $m_{e}^{*}=1.5 m_{0}$ fits best to the experimental data. The deviation of experimental data from the calculation may be duc to fluctuation of the layer thickness of the actual MQWs or other irregularity of the actual structure. However, it should be pointed out that we used the simplest model for the calculation. More accurate calculations are needed to estimate the effective mass and to predict the energy level shift exactly.

Electroluminescent (EL) diodes have also been fabricated using the multilayer structure. The EL diode consists of the multilayer structure sandwiched by indium-tin oxide (ITO) coated transparent electrode as positivc bias side and the In-containing $\mathrm{Mg}(\mathrm{Mg}: \mathrm{In})$ electrode as negative bias side. The cross-sectional view of the EL diode with the $\mathrm{Alq}_{3} / \mathrm{TPD}$ multilayer structure is shown in the inset of Fig. 4. The $\mathrm{Alq}_{3}$ layer contacts to the $\mathrm{Mg}$ :In electrode and the TPD layer to the ITO electrode. The electrode area is $2 \mathrm{~mm}$ square. The emission spectrum is shown in Fig. 4 for the EL diode of the same MQW structure with that for the PL measurement. The emission peak of the EL spectrum has appeared at around $520 \mathrm{~nm}$, whereas that of the PL spectrum at $510 \mathrm{~nm}$. This discrepancy may be explained by the difference in carrier injection as discussed in the later paragraph and by the heating of the junction during carrier injection. The emission from the TPD layer has not been observed or been very weak in the EL device. This is due to the difference in carrier injection, since carriers are excited in both layers in case of photoexcitation, whereas in the case of EL diode carriers are injected and confined mainly in the $\mathrm{Alq}_{3}$ layer due to the high energy barrier of the MQW structure.

In Fig. 5, current dependence of EL emission intensity characteristics are shown as the function of various layer thicknesses of the MQW structure. At the same injection 


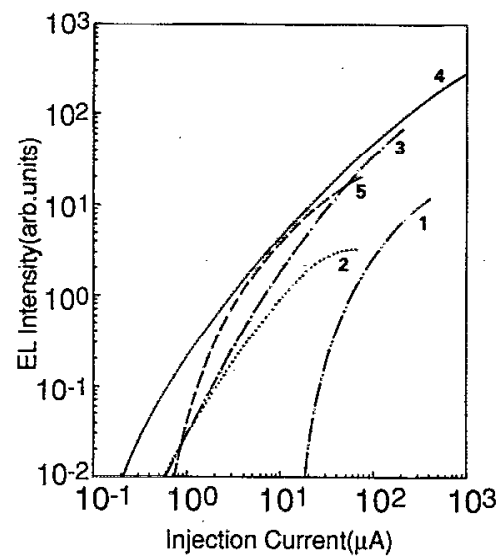

FIG. 5. Dependence of electroluminescence intensity on injection current in the diode with $\mathrm{Alq}_{3}$ /TPD multilayer structure of various layer thicknesses. $\mathrm{Alq}_{3} / \mathrm{TPD}$ multilayer thicknesses in $\mathrm{nm} .1: 1.9 / 1.9,2: 4.6 / 4.6,3$; 9.5/9.5, 4: 10.9/10.9, 5: 27.1/27.1.

current, EL intensity is the strongest at $\mathrm{Alq}_{3}$ layer thickness around $9.5-27 \mathrm{~nm}$. This is consistent, since the exciton diffusion length of the $\mathrm{Alq}_{3}$ are reported to $20 \mathrm{~nm} .^{13}$ The quantum efficiency of the EL diode with MQW structure shows the highest with the diode of $10.9 \mathrm{~nm}$ thick $\mathrm{Alq}_{3}$. This result shows that confinement of electrons in the $\mathrm{Alq}_{3}$ layer of $10-20 \mathrm{~nm}$ in thickness is most efficient for the recombination of carriers for electroluminescence.

In conclusion, the experimental results are summarized as follows.

(1) Organic multiple quantum well structure of $\mathrm{Alq}_{3}$ and TPD has been successfully fabricated using organic molecular beam deposition. The layer structure has been confirmed by low angle $x$-ray diffraction. (2) The quantum size effect has been observed in the emission from $\mathrm{Alq}_{3}$. The peak emission energy shifts to higher energy with decreasing layer thickness. (3) The EL diode has been fabricated using the multilayer structure, and the diode with a MQW structure of around $10-20 \mathrm{~nm} \mathrm{Alq}$ layer exhibited the most efficient emission characteristics.

${ }^{1}$ C. W. Tang and S. A. VanSlyke, Appl. Phys. Lett. 51, 913 (1987).

${ }^{2}$ C. Adachi, T. Tsutsui, and S. Saito, Appi. Phys. Lett. 57, 531 (1990).

${ }^{3}$ J. H. Burroughes, D. D. C. Bradley, A. R. Brown, R. N. Marks, K. Mackay, R. H. Friend, P. L. Burns, and A. B. Holmes, Nature 347, 539 (1990).

${ }^{4}$ D. Braun and A. J. Heeger, Appl. Phys. Lett. 58, 1982 (1991).

${ }^{5}$ Y. Ohmori, M. Uchida, K. Muro, and K. Yoshino, Jpn. J. Appl. Phys. 30, L1938 (1991).

${ }^{6}$ Y. Ohmori, M. Uchida, K. Muro, and K. Yoshino, Jpn. J. Appl. Phys. 30, L1941 (1991).

${ }^{7}$ G. Grem, G. Leditzky, B. Ul1rich, and G. Leising, Adv. Mater. 43, 36 (1992).

${ }^{8}$ G. Bastard, E. E. Mendez, L. L. Chang, and L. Esaki, Phys. Rev. B 26, 1974 (1982).

${ }^{9}$ J. P. van der Ziel, R. Dingle, R. C. Miller, W. Wiegman, and W. A. Nordland, Jr., Appl. Phys. Lett. 26, 463 (1975).

${ }^{10}$ Y. Ohmori, S. Tarucha, Y. Horikoshi, and H. Okamoto, Jpn. J. Appl. Phys. 23, L94 (1984).

${ }^{11}$ F. F. So, S. R. Forrest, Y. Q. Shi, and W. H. Steier, Appl. Phys. Lett. 56,674 (1990).

${ }^{12}$ F. F. So and S. R. Forrest, Phys. Rev. Lett. 66, 2649 (1991).

${ }^{13}$ C. W. Tang, S. A. VanSlyke, and C. H. Chen, J. Appl. Phys. 65, 3610 (1989).

${ }^{14}$ R. M. Fleming, D. B. McWhan, A. C. Gossard, W. Wiegmann, and R. A. Logan, J. Appl. Phys. 51, 357 (1980). 